\title{
MODELLING OF CEMENT GRINDING CIRCUITS FOR PREDICTIVE CONTROL
}

\author{
Jakob Kjøbsted Huusom*, Anker Degn Jensen* \\ Sten Bay Jørgensen*, Michael Michelsen* \\ Jørgen Knudsen **, Bodil Recke ${ }^{* *}$, John Bagterp Jørgensen *** \\ * Department of Chemical Engineering, Technical \\ University of Denmark, DK-2800 Kgs. Lyngby, Denmark \\ ** FLS Automation, Høffdingsvej 34, DK-2500 Valby, \\ Denmark \\ *** 2-Control Aps, Høffdingsvej 34, DK-2500 Valby, \\ Denmark
}

\begin{abstract}
A first principles model of a cement grinding circuit is developed for the purpose of multi-variable model predictive control (MPC). The model is based on a series of mixed flow reactors with an ideal screen to model flow between the two chambers in the ball mill. The separator is modelled by efficiency curves. Breakage of the particles is modelled as a first order process in terms of functions for the specific rate of breakage and the fragment distribution from breakage. These breakage functions are empirically based. A stationary solution for the model is found and dynamic simulations of step changes in the manipulated variables are shown. Copyright(c) 2005 IFAC.
\end{abstract}

Keywords: Modelling, nonlinear systems, comminution, grinding circuit, cement industry

\section{INTRODUCTION}

Production of cement is one of the most energy intensive industrial processes, consuming up to $2 \%$ of the worlds electricity due to several low efficiency processes. The grinding of cement clinker from the kiln is the most inefficient process in the manufacturing, with an efficiency of $1 \%$ (Benzer et al., 2001). This low efficiency makes optimization of cement clinker grinding circuits a task with large economical and environmental perspectives.

Predictive control of cement processes including the grinding circuits has been reviewed by Sánchez and Rodellar (1996). Studies of residence time distribution for the transport of charge through continuous ball mills have concluded that neither ideal mixed flow nor plug flow can model the transport satisfactorily (Austin et al., 1984). Model proposals based on a series of ideally mixed reactors or plug flow with axial dispersion have been presented in the literature in recent years for the ball mill, in order to develop model predictive control schemes. Contributions to modelling ball mills with two chambers by PDE models have been made by Boulvin et al. (1999) using grey box modelling and Lepore et al. (2003) using a reduced order PDE model with only three discrete particle sizes. Magni et al. (1999) have also proposed a simple three state ODE model for cement grinding circuits for control purposes by dynamical modelling of the holdup in the mill, the product and tailings flow rates. This model formulation does not make it possible to predict the particle size distributions in the system. Benzer et al. (2001) 
have used ideally mixed reactors to model the flow through the mill with a screen to simulate the diaphragm between the chambers in the mill. The authors successfully validated their model against data from industrial ball mills in operation.

Difficulties in modelling and controlling the milling operations arise because the grinding medium sizes are distributed over several orders of magnitude and consist of a mixture of cement clinker, gypsum and possibly a filler all with different breakage properties. It is difficult, at best, to obtain reliable measurements of key variables such as the mill hold up and the particle size distributions in the system. The size distribution of the product can be measured continuously by laser diffraction but to reduce investments this measurement is frequently provided using sieving analysis at discrete time intervals. The hold up in the mill is important in order to run the operation at the optimum efficiency. If the clinker to ball ratio is too small, energy is lost on too many ball to ball impacts, while too high a ratio cushions the impacts and risks choking the mill (Austin et al., 1984).

The characteristics for the operation presented here imply that a MPC algorithm based on a model with good ability to describe the dynamic behavior of the grinding operation could be effective. The predictive nature of this controller can compensate for the lack of continuous measurements. The use of predictive control on cement grinding circuits based on statistical models obtained by process identification has proven more efficient as compared to the more conventional fuzzy control (Knudsen, 2004).

The purpose of this study is to develop a simple first principles model description of a cement grinding circuit for control purposes. The dynamic properties of this model will be validated and parameters estimated, against plant data or a more sophisticated simulation model. Ultimately this work aims at the development of a MPC strategy for control of cement mill circuits.

In the following section a brief introduction to the system is provided and section 3 gives the model formulation of the grinding circuit including the grinding kinetics. Sections 4 and 5 discuss parameter estimation, model validation and shows dynamic simulations of the model.

\section{PROCESS DESCRIPTION}

The grinding circuit investigated here is sketched on figure 1. It consist of an F. L. Smidth UMS $46 \times 14.0$ ball mill, which is a two compartment mill with a Stanex grate diaphragm between the chambers (F.L.Smidth, 2004a). Classification is

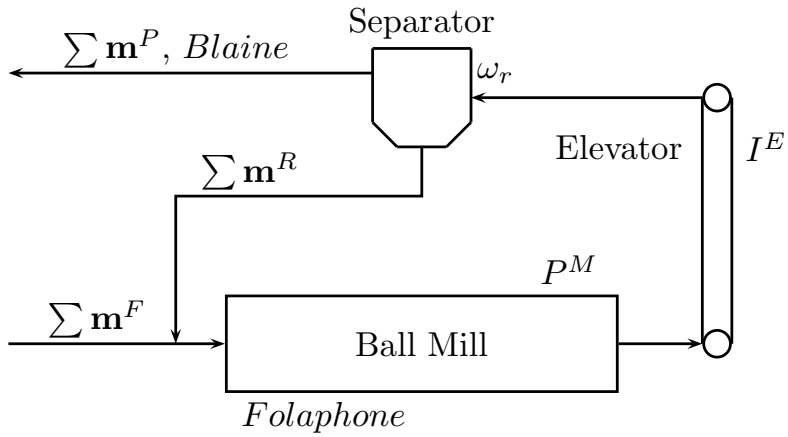

Fig. 1. The closed-loop cement grinding circuit.

performed by a F. L. Smidth SEPAX 375M-122 separator (F.L.Smidth, 2004b). The process is monitored by measuring the recycle flow rate and the rate and finness of the product. Measurements of the elevator current are proportional to the load on the carrier and the noise emission from the mill measured by a folaphone and the power consumption by the mill can be related to the hold up in the mill. Low clinker to ball ratio yields a high noise emission. Manipulated variables for this system are the feed rate and the rotor speed of the separator $\omega_{r}$.

\section{THE PROCESS MODEL FORMULATION}

The ball mill is modelled as a series of equally sized continuous, stirred tank reactors (CSTR). The first chamber consists of two reactors and the number of reactors modelling the mixing in the last chamber can vary in order to fit residence time distribution data. Here the second chamber will be modelled using three additional reactors. The flow between the chambers will be restricted by an ideal screen such that only particles smaller than a certain size can pass. The model is illustrated in figure 2 .

Particles in the system will be described by discretizing the size distribution into $n$ size intervals using sieving sizes $(z=\sqrt[4]{2})$ or a more rough discretization depending on the scaling factor $z$.

$$
\begin{aligned}
& \text { Top size of } i \text { th interval }=x_{\max }^{p}\left(\frac{1}{z}\right)^{i-1} \\
& i \in[1 ; n], \quad z \in\{\sqrt[4]{2}, \sqrt{2}, 2\}
\end{aligned}
$$

Top size particles will be labelled $x_{1}^{p}$ and the top size in this interval is $x_{\max }^{p}$. The smallest particles up till the top size of $x_{n}^{p}$ will belong to this sink interval. All particles in each size interval will be assumed to have identical properties with respect to flow and breakage. In this paper $x_{\max }^{p}=25 \mathrm{~mm}, z=2$ and $n=10$ giving a top size of $x_{n}^{p}=48.8 \mu \mathrm{m}$. 


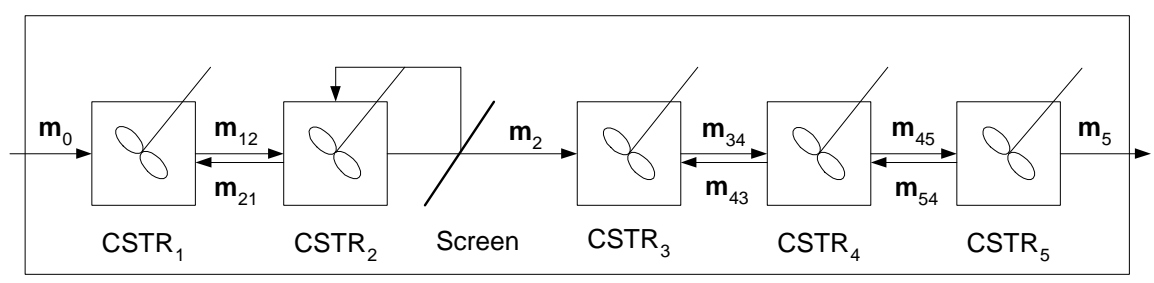

Fig. 2. A model of the cement mill consisting on five continuous, stirred tank reactors. A screen is used to model the flow between the two chambers and restrict parssing of oversizesed particles. $\mathbf{m}$ are vectors with the mass flow for each size.

\subsection{Breakage kinetics}

Breakage of particles from one size to a smaller one will be described using breakage functions $S_{i}$ for the specific rate of breakage out of size $i$ and $b_{i, j}$ for the probability that a particle that is broken out of size $j$ will be distributed to size $i$. The breakage functions $S_{i}$ and $b_{i, j}$ depends on the physico-mechanical properties of the material and the grinding conditions (Park et al., 1998). Studies by Austin et al. (1984) and Deniz (2003) show that assuming first order breakage for ball milling is often an excellent approximation. Due to the first order kinetics in the mill, a vector with the rate of formation for all the sizes can be written as

$$
\text { rate of formation }=\mathbf{K}_{\text {rate }} \mathbf{W}
$$

where the vector $\mathbf{W}$ contains the holdup of particles from size 1 to $n$. The rate matrix $\mathbf{K}_{\text {rate }}$ is constructed from the breakage functions as a lower triangular matrix.

$$
\mathbf{K}_{\text {rate }}=\left[\begin{array}{ccccc}
-S_{1} & 0 & \cdots & 0 & 0 \\
S_{1} b_{2,1} & -S_{2} & \cdots & 0 & 0 \\
\vdots & \vdots & \ddots & \vdots & \vdots \\
S_{1} b_{n-1,1} & S_{2} b_{n-1,2} & \cdots & -S_{n-1} & 0 \\
S_{1} b_{n, 1} & S_{2} b_{n, 2} & \cdots & S_{n-1} b_{n, n-1} & 0
\end{array}\right]
$$

\section{Specific rate of breakage functions}

The rate of breakage for the individual particle sizes can be approximated by a power law expression but since a small ball size is inefficient to break large lumps, Austin et al. (1984) have proposed the following expression for the rate of breakage for ball milling.

$$
S_{i}=a_{b}\left(\frac{x_{i}^{p}}{1000 \mu m}\right)^{\alpha_{b}} Q_{i} C_{2} C_{3}
$$

where

$$
Q_{i}=\frac{1}{1+\left(\frac{x_{i}^{p}}{C_{1} \mu}\right)^{\Lambda}}
$$

$\mu$ is the particle size for which $Q_{i}=0.5$ and $\Lambda$ is a positive parameter reflecting how rapidly the breakage rate decreases with particle size. Since the environment in the ball mill is not uniform or static, a set of scale up and correction factors, (4), are multiplied in equation (3) to account for changes in e.g. ball size and hold up.

$$
\begin{aligned}
C_{1} & =\left(\frac{d}{d_{T}}\right)^{2} \\
C_{2} & =\left(\frac{d_{T}}{d}\right)^{N_{0}} \\
C_{3} & =\frac{1+6.6 J_{T}^{2.3}}{1+6.6 J^{2.3}} \exp \left(-c\left(U-U_{T}\right)\right)
\end{aligned}
$$

The subscript $T$ refers to a set of reference conditions and $d, J$ and $U$ are the ball diameter, fraction of balls filling by volume in the mill and void fraction between balls, filled with particles for a mill at rest respectively.

A similar approach has been used by Sato et al. (1996) where the authors relates the material dependent parameter $a_{b}$ in (3) to the Hardgrove grindability index, $H G I$, as follows:

$$
a_{b}=1.5\left(1-\exp \left(-0.0026 H G I^{1.5}\right)\right)
$$

and the exponet $\alpha_{b}$ was found to 1.25.

\section{Breakage distribution functions}

Austin et al. (1984) have presented (6) as an empirical correlation for the cumulative breakage distribution function where $b_{i, j}=B_{i, j}-B_{i+1, j}$ for $i<n$.

$$
B_{i, j}=\Phi_{j}\left(\frac{x_{i-1}^{p}}{x_{j}^{p}}\right)^{\gamma}+\left(1-\Phi_{j}\right)\left(\frac{x_{i-1}^{p}}{x_{i}^{p}}\right)^{\beta}
$$

where

$$
\begin{aligned}
\Phi_{1} & =\frac{1-\left(\frac{1}{z}\right)^{\beta}-b_{2,1}}{\left(\frac{1}{z}\right)^{\gamma}-\left(\frac{1}{z}\right)^{\beta}} \\
\Phi_{j+1} & =\Phi_{j}\left(\frac{1}{z}\right)^{-\delta_{N}}
\end{aligned}
$$

and $z$ is the same as used in equation (1). The parameters $\gamma$ and $\beta$ are related to the distribution of fragments as shown on figure 3 . The parameter $\delta_{N} \approx 0.2$ for cement clinker (Austin et al., 1984). 


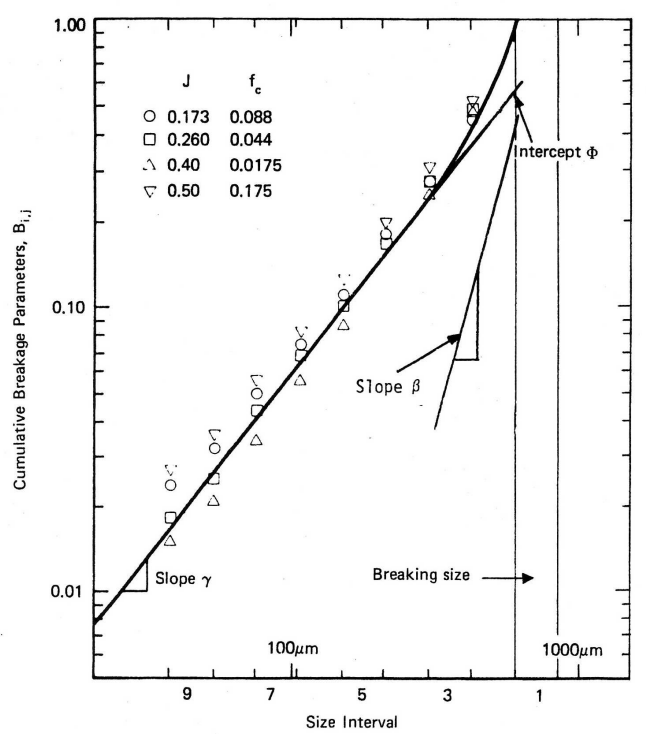

Fig. 3. Cumulative fragment distribution from breakage of the top size interval, $x_{i=1}^{p}$, of a sample of quartz under changing ball and charge filling. (Austin et al., 1984, page 87)

\subsection{Mass balances}

Given the formulation in terms of well mixed reactors the mass balances for the system becomes.

$$
\begin{aligned}
& \frac{d \mathbf{W}_{1}}{d t}=\mathbf{m}_{0}+\mathbf{m}_{21}-\mathbf{m}_{12}+\mathbf{K}_{\text {rate }, 1} \mathbf{W}_{1} \\
& \frac{d \mathbf{W}_{2}}{d t}=\mathbf{m}_{12}-\mathbf{m}_{21}-\mathbf{m}_{2}+\mathbf{K}_{\text {rate }, 2} \mathbf{W}_{2} \\
& \frac{d \mathbf{W}_{3}}{d t}=\mathbf{m}_{2}+\mathbf{m}_{43}-\mathbf{m}_{34}+\mathbf{K}_{\text {rate }, 3} \mathbf{W}_{3} \\
& \frac{d \mathbf{W}_{4}}{d t}=\mathbf{m}_{34}+\mathbf{m}_{54}-\mathbf{m}_{43}-\mathbf{m}_{45}+\mathbf{K}_{\text {rate }, 4} \mathbf{W}_{4} \\
& \frac{d \mathbf{W}_{5}}{d t}=\mathbf{m}_{45}-\mathbf{m}_{54}-\mathbf{m}_{5}+\mathbf{K}_{\text {rate }, 5} \mathbf{W}_{5}
\end{aligned}
$$

where $\mathbf{m}_{0}$ is given by the feed to the mill and all the mass flows from reactor $i$ to the $j$ 'th, $\mathbf{m}_{i j}$, is first order functions of the hold up in the $i$ 'th reactor as shown in equation (9a) and not depending on the conditions in reactor $j . \mathbf{m}_{2}$ and $\mathbf{m}_{5}$ are given as first order functions of the particle hold up in the chamber the particles are flowing from.

$$
\begin{aligned}
\mathbf{m}_{i j} & =k_{1} \mathbf{W}_{i} \\
\mathbf{m}_{2} & =q_{1} \frac{(\mathbf{I}-\mathbf{R}) \mathbf{W}_{2}}{\sum(\mathbf{I}-\mathbf{R}) \mathbf{W}_{2}}
\end{aligned}
$$

where

$$
\begin{aligned}
q_{1} & =0.0171 \sum_{\text {Chamber } 1} \mathbf{W}-0.0981 \\
\mathbf{m}_{5} & =q_{2} \frac{\mathbf{W}_{5}}{\sum \mathbf{W}_{5}}
\end{aligned}
$$

where

$$
q_{2}=0.00705 \sum_{\text {Chamber } 2} \mathbf{W}-0.0717
$$

The matrix $(\mathbf{I}-\mathbf{R})$ gives a diagonal matrix which elements are the fraction of a particle size that are transported thourgh the diaphragm. Since the diaphragm is model as an ideal screan these elements does only take the values 0 or 1 . The restrictions on the flow in the mill expresses that the flow between two of the reactors inside a mill chamber in the model is driven by the difference in hold up. The flow through the diaphragm in the middle is only occurring from the first to the second chamber.

\subsection{Classification}

The separator will be described by a static model since it is assumed to have much faster dynamics than the ball mill. The model consists of a efficiency curve (Tromp curve) giving the fraction of accepted particles, $w_{i}^{p}$, as a function of particle size. These particles leaves with the product flow. Separators like the F. L. Smidth SEPAX 375M122 separator has Tromp curves which exhibit a "fish hook" shape and a small amount of the feed to the separator is bypassed without classification (F.L.Smidth, 2004b). Benzer et al. (2001) uses a Tromp curve with the desired properties.

$$
w_{i}^{p}=\left(1-\delta_{b p}\right) \frac{\left(1+\beta_{1} \beta_{2} \frac{x_{i}^{p}}{d_{50}}\right)\left(\exp \left(\alpha_{T}\right)-1\right)}{\exp \left(\alpha_{T} \beta_{2} \frac{x_{i}^{p}}{d_{50}}\right)+\exp \left(\alpha_{T}\right)-2}
$$

$$
i \in\{1,2, . ., n\}
$$

where $\delta_{b p}$ is the bypass factor and $\beta_{1}$ is the fish hook parameter. The parameter $\beta_{2}$ determine the location for the cutsize $d_{50}$. Variation in the air speed, $v_{a i r}$, and the rotor speed, $\omega_{r}$, in the separator are incorporated in the Tromp curve through the cut size and the sharpness factor $\alpha_{T}$.

$$
\begin{aligned}
& d_{50}=f\left(v_{a i r}, \omega_{r}\right) \\
& \alpha_{T}=g\left(v_{\text {air }}, \omega_{r}\right)
\end{aligned}
$$

Choosing $\beta_{1}=0$ in equation (10) removes the hook shape for the efficency curve.

\section{ESTIMATION AND VALIDATION}

The model for the circuit containes a number of known and unknown parameters. Known parameters are the dimensions of the equipment and the properties of the feed. The unknown parameters can in some cases be determined from independent experiments and have been reported in literature (Austin et al., 1984; Sato et al., 1996). For parameter estimation a selection of parameters 
representing the transport through the mill, the grinding and the separation have been chosen

$$
\boldsymbol{\theta}=\left[\begin{array}{lllll}
k_{1} & \alpha_{b} & b_{21} & \beta_{2} & C_{b}
\end{array}\right]^{T}
$$

where the parameter $C_{b}$ is a shape factor correcting for the non spherical appearance of the product.

The parameter estimates are produced by minimizing a weighted least squares objective function based on simulated steady state data from a sophisticated simulation model, the CEMulator, developed by FLS-Automation A/S for operator training and test of control systems (Jensen, 2004). The inputs for these static solutions have been chosen to span the operational space with respect to feed composition, mill feed rate and the angular velocity of the rotor in the separator. The validation criterion for the model is to reduce the systematic errors in the residuals between the prediction from the two models. Further more dynamic responses have been simulated to illustrate how the gains in the simple mixed flow model resembles step responses from the more comprehensive CEMulator. Estimation based on simulated outputs provide information on how the system behaves under ideal circumstances without noise and assuming access to all measurements online. These investigations have revealed strong correlations between some parameters. The mixed flow model have shown potential to predict the dynamic behavior from the CEMulator with an offset error but this work have not been completed at this stage and estimation using plant data have not been attempted.

\section{PROCESS SIMULATIONS}

The process model has been implemented and simulated in MATLAB(R). A stationary solution is found by solving equation (8) with the time derivatives equal to zero. The composition of the feed is $80 \%$ clinker, $15 \%$ gypsum and $5 \%$ filler with a size distribution. The initial model parameters are based on preliminary estimations, and the feed size distributions are presented in Huusom (2005).

Figure 4 shows the normalized cumulative size distribution of the feed, recycle and product flows at the stationary operation point. Particles belonging to the two largest size intervals are not able to enter the second chamber in the mill through the screen hence these sizes are not present in the feed to the separator. The figure shows that the parameters used in the simulation causes an effective grinding in the mill reducing the particle size below $1 \mathrm{~mm}$. Given a mill in steady state operation some step changes has been simulated in the manipulated variables in order to investigate

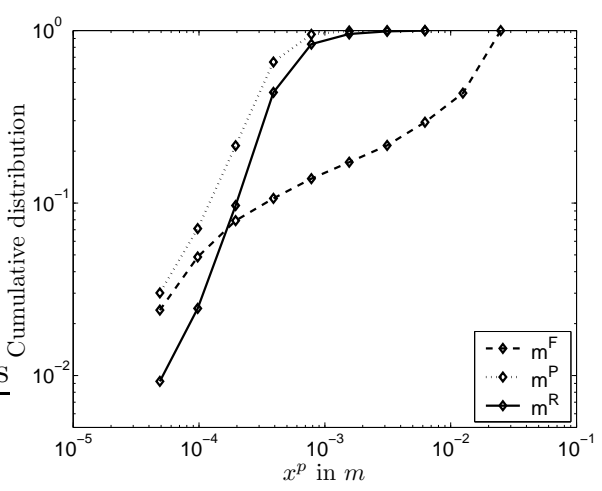

Fig. 4. Normalized cumulative size distribution of the mill feed, $\mathbf{m}^{F}$, tailings, $\mathbf{m}^{R}$ and product flow, $\mathbf{m}^{P}$.

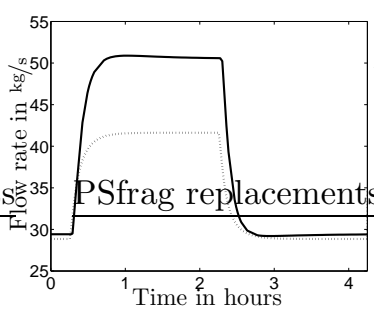

(a) Reject flow rate

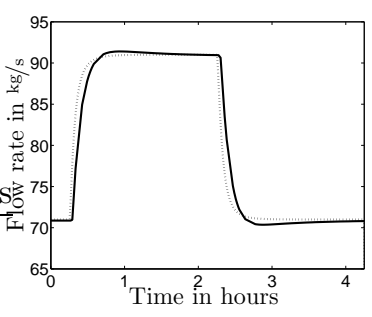

(b) Product flow rate
Fig. 5. Simulation of step responses in recycle and product flow rate for a 20 tonne/hour changes in the feed rate to the system. The full line is the reference response from the CEMulator and the dotted line is the simulation with the mixed flow model.

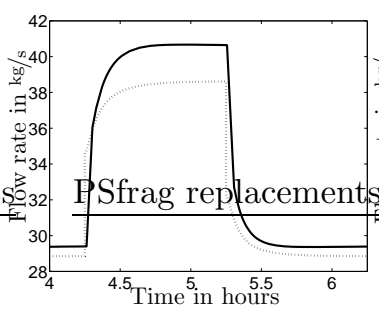

(a) Reject flow rate

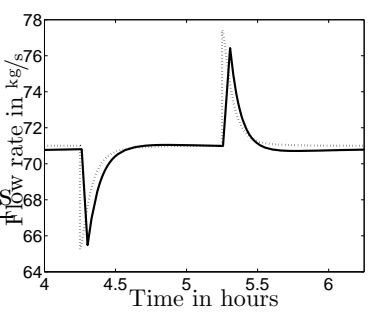

(b) Product flow rate

Fig. 6. Simulation of step responses in recycle and product flow rate for $30 \%$ change in the angular velocity of the rotor in the separator. The full line is the reference response from the CEMulator and the dotted line is the simulation with the mixed flow model.

the dynamic behavior of the system. An increase in the feed rate to the circuit has been introduced after 15 minutes simulation and the actuator is again given its initial value after the transient has settled. After four hours simulation a step in the angular velocity of the separator rotor has been introduced and after an one hour transient the system is brought back to the initial state. Here 
only the response for the two flow rates from the separator are shown of the six process outputs. Figure 5 shows the step response of the flow rate for the product and recycle together with a reference response simulated with the CEMulator model. Figure 6 shows the corresponding responses for a step change in the angular velocity. These figures show that the system is faster to return to a steady state solution given a change in the separator speed compared to changes in the feed rate. The figure also reveals that the estimated set of parameters gives accurate predictions for the dynamic response in the product flow but that the gain for the reject flow rate is estimated with an offset. It is seen that an increase in the feed rate produces a general increase in the flows of the system while a change in the separation does not change the static value for the production rate but only the size distributions in the system and the recirculation ratio. On figure 6 it is observed that the separator in the CEMulator has a dynamic and not an instant transition when the step is applied but that the modelling of the separation using a static model is a reasonable simplification. The step responses of the product and recycle flow for a change in the separator speed resembles responses in the PDE-model by Boulvin et al. (1999).

\section{CONCLUSION}

A nonlinear process model for the cement grinding circuit has been developed and presented. It consists of a mixed flow model for the ball mill and an efficiency curve for the separator. Comminution of particles is modelled using a first order rate expression and an empirical correlation for the specific breakage rate and the breakage distribution functions.

Given a set of model parameters, a steady state solution has been found for the circuit. The $\mathrm{cu}-$ mulative particle size distributions for the feed, product and recycle flows are presented for this solution. The parameters have been estimated based on simulated static data.

The dynamic responses show that changes in the separator speed controls the flow rate of rejected particles and the fineness of particles in the product flow while changes in the feed rate change the production rate of the grinding system. The responses are similar compared to other step responses in the literature.

The model have not yet been validated on experimental data and further work has to be done to reduce the systematic errors in the residuals. The mixed flow model has shown potential to predict the dynamic behavior of the reference model despite a far simpler structure.

\section{REFERENCES}

Austin, L. G., R. R. Klimpel and P. T. Luckie (1984). Process Engineering of Size Reduction: Ball Milling. Society of Mining Engineers.

Benzer, H., L. Ergun, A. J. Lynch, M. Oner, A. Gunlu, I. B. Celik and N. Aydogan (2001). Modelling cement grinding circuits. Minerals Engineering 14(11), 1469-1482.

Boulvin, M., C. Renotte, A. Vande Wouwer, M. Remy, S. Tarasiewicz and P. Csar (1999). Modeling, simulation and evaluation of control loops for a cement grinding process. European journal of control 5, 10-18.

Deniz, V. (2003). A study on the specific rate of breakage of cement materials in a laboratory ball mill. Cement and concrete research 33, 439-445.

F.L.Smidth (2004a). Ball mills type UMS, TMS and TUMS. Brochure.

F.L.Smidth (2004b). SEPAX separator. Brochure. Huusom, Jakob Kjøbsted (2005). Modelling of cement mills. Master's thesis. Technical university of Denmark.

Jensen, Torsten Vagn (2004). User Manual CEMulator. 7 ed.. FLS-Automation A/S. Høffdingsvej 34, DK-2500 Valby, Denmark.

Knudsen, Jørgen K. H. (2004). Model predictive control of a cement mill. In: Dansk kemiingeniørkonference 2004. pp. 59-60.

Lepore, R., A. Vande Wouwer and M. Remy (2003). Nonlinear model predictive control of cement grinding circuits. In: ADCHEM2003.

Magni, L., G. Bastin and V. Wertz (1999). Multivariable nonlinear predictive control of cement mills. IEEE. Transactions on control systems technology 7(4), 502-508.

Park, Jai Koo, Young Jeong, Jeong Il Yang and Moon Young Jung (1998). Grinding time for control of the size fraction of products in the attrition milling. Korean J. Chem. Eng. 15(4), 375-380.

Sánchez, Juan M. Martin and José Rodellar (1996). Adaptive Predictive Control. From the Concepts to Plant Optimization.. Prentice Hall.

Sato, K., N. Meguri, K. Kanemoto, T. Hasegawa and T. Maruyama (1996). Breakage of coals in ring-roller mills part 1 . the breakage properties of various coals and simulation model to predict steady-state mill preformance. $P_{O}$ wder Technology 86, 275-283. 